\title{
Editorial
}

\section{Kinderfernsehen als Thema öffentlicher Debatten: eine Einordnung in ein kulturtheoretisches Modell}

\author{
Ben Bachmair
}

\section{Wie wurde Kinderfernsehen bisher zum Thema?}

Der Aufruf, Kinderfernsehen wieder zum Thema zu machen, bezieht sich auf zwei neuere Entwicklungsphasen ${ }^{1}$, in denen Kinderfernsehen Thema war. Das war einmal in den 1970er Jahre, als sich in der Phase des Vorschulbooms eine öffentliche Debatte mit Kinderfernsehen wie «Sesame Street», «Rappelkiste» und der «Sendung mit der Maus» beschäftigte. Damals, Anfang der 1970er Jahre wurde Kindheit neu gedacht und auch das Verhältnis zum gesellschaftlich dominanten Medium Fernsehen kontrovers diskutiert. ${ }^{2}$ Sendungen wie die mit der Maus wollten nicht weniger, als mediengerecht in das Verhältnis der Kinder zur Welt eingreifen und sich dabei auf die Seite der Kinder stellen, um ihnen die Welt in ihrer Sichthöhe erklären. Sendungen wie die Sesamstrasse entstanden in der Absicht, Kinderfernsehen als Lernressource einzusetzen, um sozialkulturelle Defizite zu kompensieren. 20 Jahre später, in den 1990er Jahren, gingen Impulse, sich mit Kinderfernsehen auseinander zu setzen, nicht von den sozialkulturellen Umbrüchen wie Individualisierung aus, sondern von der Ausgliederung der Kinderprogramme aus den Vollprogrammen der öffentlich-rechtlichen Sender, die zur Gründung des KiKa führte. In diesem Kontext entstand 1995 eine «Debatte zum Kinderfernsehen» in der Form eines «Runden Tisches», den die Medienstellen der beiden grossen Kirchen moderierten. Der Anstoss dazu und die Dynamik dieses «Runden Tisches Qualitätsfernsehen für Kinder» kam vom Herausgeber dieses Heftes in Zusammenarbeit mit Paul Löhr, dem damaligen Leiter des Internationalen Zentralinstituts für das Jugend- und Bildungsfernsehen des Bayerischen Rundfunks, Dr. Uwe Rosenbaum, damals Direktor des SWR-Senders in Mainz und Vorstandmitglied des Fördervereins «Deutscher Kinderfilm» sowie Ludger Verst, damals Leiter des Referats Rundfunk der Zentralstelle Medien der Deutschen Bischofskonferenz und Mechthild Appelhoff von der Nordrheinwestfälischen Landesmedienanstalt. An der Debatte in Form gemeinsamer Treffen nahmen die für das Thema verantwortlichen Vertre-

\footnotetext{
Überblick über die Entwicklung des Kinderfernsehens finden sich bei Schmidbauer 1987, Erlinger u. a. 1995, Erlinger, Stötzel 1991.

2 Einen informativen Zugang bietet Paus-Haase 1995. Schäfer, 1995, gibt einen Überblick über die TV-Programmentwicklung.
} 
ter der öffentlich-rechtlichen und der privaten Sender teil. Ein Sammelband mit vor allem wohl abgewogenen Meinungen erschien 1998 unter dem Titel «Debatte Kinderfernsehen» (Zentralstelle Medien der Deutschen Bischofskonferenz, Gemeinschaftswerk der Evangelischen Publizistik 1998).

Die «Thesen» dieses «Runden Tisches: Qualitätsfernsehen für Kinder» lesen sich heute wie die rückblickende Zusammenfassung des Programms innovativen Kinderfernsehen der 1970er Jahre mit der schon genannten Sendung mit der Maus oder der Sesamstrasse als Ausdruck eines neuen Kinderbildes, in dessen Mittelpunkt sich eigenständig entwickelnde Kinder stehen.

\section{«Thesen des Runden Tisches: Qualitätsfernsehen für Kinder» von 1995}

Der «Runde Tische» verabschiedete seine These am 4. November 1996 in Mainz und veröffentlichte sie später auch als Teil des «Programmberichts des Runden Tisches ¿Qualitätsfernsehen für Kinder»» (Zentralstelle Medien der Deutschen Bischofskonferenz, Gemeinschaftswerk der Evangelischen Publizistik 1998, S. 16 f.)

\section{Präambel}

Fernsehen spielt im Alltag der Heranwachsenden, für ihre Entwicklung, Lebensorientierung und Daseinsbewältigung eine massgebliche Rolle. Kindheit ist heute mediatisierte Kindheit in einer unübersichtlichen Welt. Den Programmverantwortlichen, aber auch den gesellschaftlichen Gruppen, Eltern, Erziehern und Politikern kommt daher im Blick auf das Fernsehen besondere Verantwortung zu. Das Kinderprogramm in Deutschland bedarf angesichts aktueller Programmentwicklungen sowie neuer technologischer Verbreitungs- und Nutzungsmöglichkeiten einer übergreifenden, fördernden Initiative.

Die am Runden Tisch beteiligten Sender, Produzenten, Gruppen, Verbände und Kirchen fordern daher dazu auf, Kinderprogramme durch die Einhaltung vereinbarter Standards zu fördern, sie kontinuierlich und öffentlich zu diskutieren und zu Vielfalt und Qualität zu ermutigen.

Sie vereinbaren folgende Thesen zum Kinderfernsehen:

1. Kinder haben einen Anspruch auf qualitativ hochwertige Fernsehprogramme, die den professionellen und ästhetischen Standards anderer Programme entsprechen.

2. Kinderprogramme bieten eine Vielfalt von Genres, Formaten und Inhalten. Sie bereiten diese in Kindern angemessener dramaturgischer Form auf und ermöglichen Innovation und Originalität.

3. Kinderprogramme unterhalten und informieren. Sie nehmen die Bedürfnisse und Erwartungen, Hoffnungen und Neugier der Kinder ernst. Sie berücksichtigen die Erfahrungen und Erlebnisse der Kinder und bieten ihnen Orientierungshilfen. Sie geben ihnen Raum für eigene Gefühle, machen ihnen Mut, vermitteln Stärke und helfen ihnen, sich altersgerecht zu entwickeln. 
4. Kinderprogramme nehmen die Fragen, Themen und Stoffe von Kindern ernst. Sie berücksichtigen alters- und geschlechtsspezifische Unterschiede, fördern das Bewusstsein der Kinder für die eigene und für fremde Kulturen und unterstützen deren Wertschätzung.

5. Kinderprogramme werden zu verlässlichen Zeiten gesendet. Die Platzierung der Programme muss altersangemessen und für Kinder leicht auffindbar sein.

6. Kinderprogramme erfordern eine gute finanzielle Ausstattung sowie qualifizierte Programmmitarbeiterinnen und -mitarbeiter. Dies setzt unterschiedliche Formen der Finanzierung voraus.

\section{Von der legitimierenden Rückschau auf eine erfolgreiche Innovation zur konservierenden Kritik}

Vorausschauend waren diese Grundsätze nicht, statt dessen formulierten sie in grosser Ausgewogenheit, was sich in den 20 Jahren davor als Erfolgsgeschichte des Kinderfernsehens bewährt hatte. Das war vor allem, Kinderfernsehen als Teil der Kinderkultur zu produzieren und zu bewerten, dabei das eigenständige Kind als Mediennutzer erst zu nehmen. Nun, die zurückblickende Legitimation einer kulturellen Innovation ist sicher hilfreich, insbesondere wenn in dieser Innovationslogik auch der Spartenkanal KiKa zum Erfolg wurde. Etwa 10 Jahre später, Mitte 2007, berichtet der Medienjournalist Tilmann Gangloff von der aktuellen kritischen Diskussion im Tenor des «Runden Tisches: Qualitätsfernsehen für Kinder», jetzt mit dem Schwerpunkt der «finanziellen und kreativen Krise» (Süddeutsche Zeitung Nr. 174, S. 15, 31. Juli 2007). Gangloff schreibt von der kritischen Erörterung, dass öffentlich-rechtlichen Wissensendungen sich nicht dem Zeichentrick unterzuordnen hätten und vom Ruf nach der Pflege der «europäischen Tradition» von Zeichentrick und Animation, wozu u. a. das Land Nordrheinwestfalen im Rahmen des Grimme-Preises einen Sonderpreis für «Produktionen zur spezifischen kulturellen Bildung von Kindern» ausgelobt hat.

Angesichts des kulturellen Wandels von Kindheit ist solch ein Verharren auf der Qualitäts-Diskussion von Mitte der 1990er Jahre nun alles andere als eine rückblickende Legitimation der Integration des Kinderfernsehens in den gelungenen kulturellen Wandel von Kindheit. Jetzt werden wichtige Teile der aktuellen gesellschaftlichen Wirklichkeit ausgeblendet wie die Fragmentierung der Kinderkultur mit Kindern, die in prekären sozioökonomischen Lebenslagen leben und ihre eigenständigen kulturellen Praktiken entwickeln (vgl. Paus-Hasebrink et al. und Mikos et al. in diesem Heft, Milieu spezifische Fernsehnutzung bei Kuchenbuch 2003). Zudem ist zu fragen, wo die Diskussion des laufenden radikalen Wandels der Massenkommunikation stattfindet, der weg führt von redaktionell verantworteten, editierten Programme und die die von den Nutzer generierten Programmen auf Internet-Plattformen wie YouTube sowie individualisierte Zugänge mit dem multimodalen Handy in den Mittelpunkt der Massenkommunikation stellt. 
Ein kulturtheoretisches Modell zur Klärung des Diskurses zum Kinderfernsehen Mein Vorwurf, diese Diskussion führe an den wesentlichen aktuellen Themen vorbei, indem sich die Debatte auf Qualität fokussiert, bekommt leicht die Schlagseite zur Besserwisserei. Deshalb will ich den Versuch machen, Kinderfernsehen in das von Anthony Giddens (1984) entwickelte Schema zur Beschreibung der Beziehung gesellschaftlicher Strukturen und handelnder Menschen (Agency) einzuordnen. Dieses bipolar angelegte Model der Strukturierung (structuration) erweitere ich zu einer argumentativen Dreiecksrelation, indem ich die kulturellen Praktiken zum Fernsehen als konstitutiv für Kinderfernsehen mit berücksichtige.

Mit der Argumentationsfigur «Struktur/Handlungsweisen und Handlungskompetenzen/kulturelle Praxis> schliesse ich mich also dem wissenschaftlichen Diskurs an, den Antony Giddens, er ist der prägende britische Kultursoziologe, in den 1980er Jahren mit der dialektischen Beziehung von Structure und Agency begann. Diese Diskussion gelangte über die Cultural Studies in die Medienpädagogik. Beim Konzept der kulturellen Praxis folge ich u. a. Stuart Hall (1997, S. 36), dem bestimmenden Theoretiker der britischen Cultural Studies oder dem amerikanischen Sozialsemiotiker und Pädagogen Etienne Wenger (1998 u. a. S. 5.), der diesen Gedanken für die Analyse von Lernkulturen nutzt.

In dieser Denkrichtung lässt sich Kinderfernsehen als eingespannt in eine Dreiecksbeziehung von

- Strukturen der Medien und der Kindheit,

- Akteuren, das sind vor allem die Kinder bzw. die Jugendlichen und ihre Eltern,

- Alltagspraxis diskutieren.

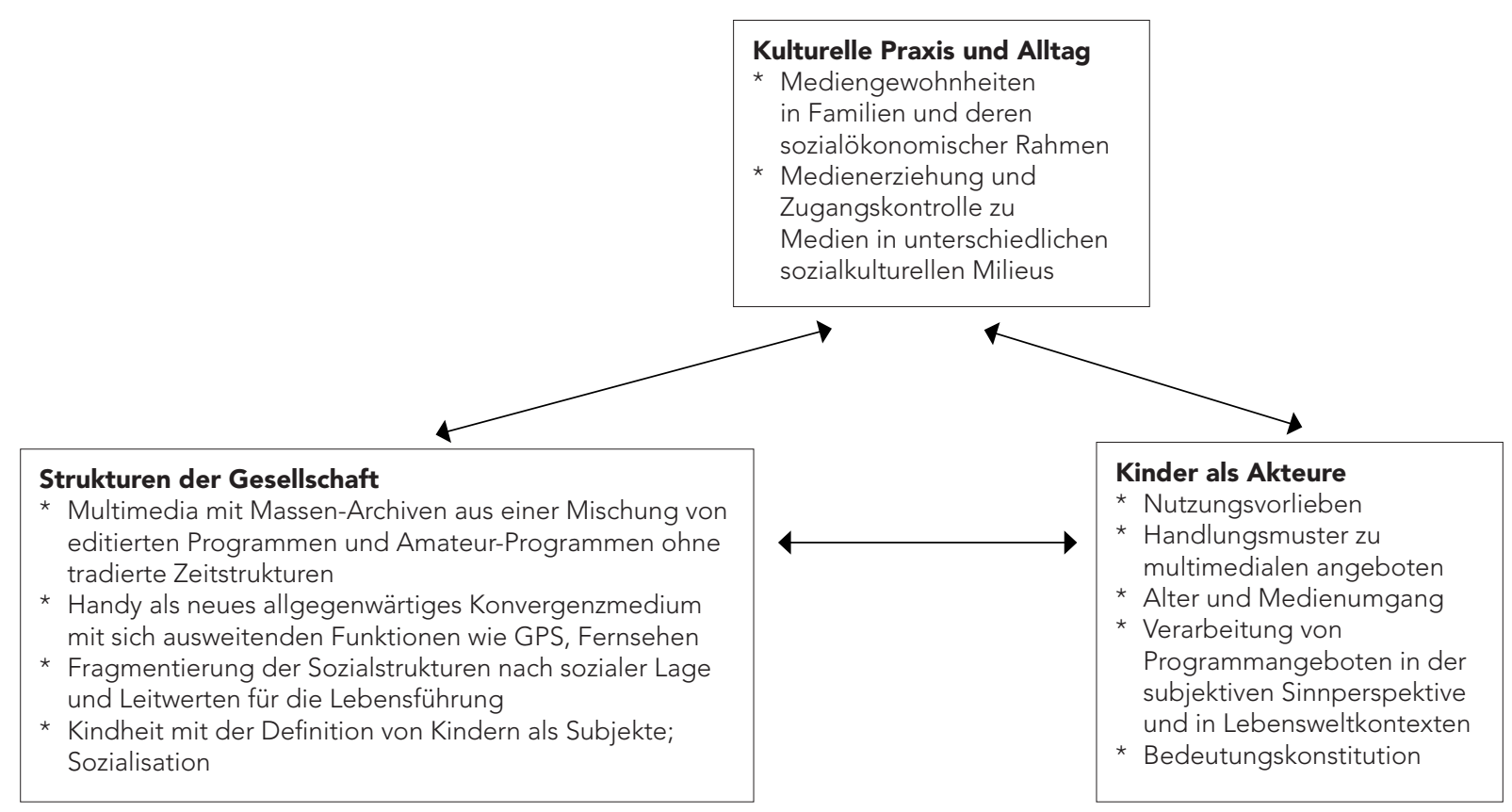


Wenn ich weiter oben die Qualitätsdiskussion des Runden Tisch Qualitätsfernsehen für Kinder der Mitte der 1990er Jahre als Legitimation einer veränderten Definition von Kindheit, Subjektivität und Sozialisation beschrieben habe, dann steht dabei der gesellschaftliche Strukturwandel der 1970er Jahre mit einer neuen Definition von Subjektivität und Lebensgestaltung im Vordergrund. Diese Debatte Qualität liest sich trotz der deutlichen Ausrichtung auf Kindheit als einer gesellschaftlichen Struktur nicht als Erörterung des damals aktuellen Strukturwandels in Richtung einer Individualisierung und Fragmentierung von Lebenswelten. Statt dessen betonte diese Debatte die erfolgreiche Ausrichtung des Kinderfernsehens auf das neue Kindheitsmodell, in dessen Mittelpunkt Kinder stehen, die in ihrer Lebenswelt und Entwicklungsperspektive Bedeutung und Sinn auch mit Medien schaffen. Der gesellschaftliche und mediale Strukturwandel, wie ich ihn in der damaligen Diskussion versuchte zu konturieren (Bachmair 1998), war noch kein Thema und ging nicht vorausschauend in die Leitlinie für Kinderfernsehen ein.

\section{Schwerpunkte der Diskussion des vorliegenden Themenheftes}

Die mit diesen strukturellen gesellschaftlichen Veränderungen einher gehenden Verwerfungen greift der Beitrag von Schwarb, Signer und Bonfadelli heute empirisch auf:

Die quantitative Programmanalyse weist nach, dass das Kinderangebot im Schweizer Fernsehen seit 1980 absolut zugenommen hat. (S. 18). Zudem fällt auch auf, dass die Beobachter - zum Teil inspiriert aus früheren Zeiten - stark inhaltlich-pädagogisch und aus einem Anspruch an ein öffentliches Medium heraus argumentieren, während die Macher/innen stärker Fragen der Umsetzung (formal) und der Resonanz im Publikum (Publikumszuwendungen, Einschaltquoten) gewichten. Letzteres ist auch ein Hinweis darauf, wie herausfordernd es für Kinderprogrammschaffende insgesamt sein muss, in dynamischen Medien- und Lebenswelten von heutigen Kindern überhaupt Resonanz zu erzeugen. (S. 20)

Heute ist der Strukturwandel der Medien mit dem Stichwort Konvergenz in der Diskussion der Sender angekommen. Auf meine Bitte um eine Stellungnahme zum Thema Kinderfernsehen, mit der ich bei der Vorbereitung dieses Themenheftes an die Redaktionen der für Kinder relevanter deutschen Fernsehsender herangetreten war, ging Claude Schmit, Geschäftführer von SuperRTL explizit auf diese medienstrukturelle Entwicklung ein. Bezogen auf das Argumentationsschema von Struktur, Akteuren und kultureller Praxis erörtert er die multimodale und multimediale Entwicklung der Massenkommunikation als wesentliche Veränderung gesellschaftlicher Strukturen. 
Derzeit entstehen ganz neue Medienformen. Schlagworte wie Konvergenz, user generated content, Digitalisierung des Fernsehens und interaktive Web 2.0-Anwendungen wie Blogs, Podcasts und Newsfeeds machen die Runde. Insbesondere das Internet bietet dem Einzelnen die Möglichkeit, den Rezipienten-Status zu überwinden und aktiv an der Mediengestaltung teilzunehmen. Virtuelle Communities wie MySpace verzeichnen stetig wachsende Mitgliederzahlen, Videoplattformen wie YouTube werben mit dem Slogan «broadcast yourself». Hier entsteht eine völlig neue Mediennutzungskultur, die die bestehende Fernsehlandschaft radikal umwälzen kann. Doch inwieweit sind diese neuen Medien bereits Bestandteil des Medienalltags von Kindern? Wie reagieren wir als Fernsehsender auf diese Entwicklungen? Und welche Implikationen ergeben sich daraus für die Medienpädagogik? (Claude Schmit, in diesem Heft S. 1)

Mikos und Töpper stellen sich einem Teil dieser Fragen, indem sie nach den Handlungsformen der Kinder in bezug auf Wissen und deren Stellung im Alltag von Mediengeboten, Schule und Familie fragen. Dabei finden sie unter anderem «unterschiedliche Nutzertypen (S. 10). «Leider» so stellen Mikos und Töpper fest, bleibt das vom Fernsehen erworbene Wissen isoliert und fragmentarisch, weil sie

offenbar nur unzureichend von anderen Institutionen wie dem Elternhaus und noch weniger der Schule aufgegriffen werden. Tiefgehenderes erfahren sie nur dann, wenn Wissenssendungen, soziales Umfeld oder Schulunterricht zufällig eines dieser Themen aufgreifen und so den Wissensdurst der Kinder stillen können. Insbesondere Kindern aus geringeren Bildungsmilieus fehlen anscheinend entsprechende Anregungen aus dem sozialen Umfeld, um durch (Kinder-)Fernsehen erworbenes Wissen oder Anregungen sinnvoll in ihren lebensweltlichen Kontext zu integrieren. (S. 12)

Damit verweisen Mikos und Töpper auf die sich verändernden Sozialstruktur. Mir erscheint hierbei wichtig, dass heute in die «neues Sozialstruktur vorgegebene sozioökonomische Lebenslagen und wählbare Lebensorientierungen und Werte eingehen, die zu sozialkulturellen Milieus mit spezifischen Mediennutzungsmustern und Lernmustern führen. In diesen Milieus mit ihren spezifischen Mediennutzungs- und Lernmuster kann Kinderfernsehen wirksam werden. Dabei spielt eine generelle und die Sozialmilieus übergreifende Qualitätsdefinition nur eine untergeordnete Rolle.

Diesem Zusammenhang widmen sich explizit Paus-Hasebrink, Bichler und Wijnen mit der «Milieuzugehörigkeit der Kinder als Einflussfaktor in bezug auf ihren Umgang mit Medien und den daraus resultierenden Auswirkungen auf ihre Sozialisati- 
on - und dies speziell in sozial schwächeren und anregungsärmeren Milieus.» Hier, so eines der Ergebnisse der österreichischen Untersuchung, ist die Erziehungsund Mediennutzungspraxis der Eltern wichtig, die sich mit den neuen massenmedialen Strukturen verbindet:

Crossmedial vermarktete (Zeichentrick-)Sendungen von privaten TV-Anbietern, die im Rahmen der ausgewiesenen Kinderprogramme ausgestrahlt werden und vornehmlich der Klassifikation «Kindermarke» zuzuordnen sind, zählen ... zu den favorisierten Angeboten bei den fünf- bis sechsjährigen Kindern aus sozial benachteiligten Familien. (S. 7 f.)

Im Bereich der kulturellen Praktiken stellen Paus-Hasebrink et al. eine «Unausgewogenheit» der «familialen Medienerziehung» in sozial benachteiligten Familien fest:

Die Eltern verurteilen demnach zwar einerseits bestimmte Fernsehangebote als nicht kindgerecht, lassen ihre Kinder jedoch andererseits genau solche Inhalte und Sendungen rezipieren.

Die Unausgewogenheit ... zeigt sich auch darin, dass es den zumeist allein erziehenden Müttern und Vätern einerseits wichtig ist, dass ihre Kinder Medien adäquat bedienen und die Funktionsweise von Medien verstehen können, sie es jedoch andererseits im Regelfall an (kommunikativen) Hilfestellungen fehlen lassen. Medienbezogene Gespräche zwischen Eltern und Kindern finden nur sporadisch, zumeist während der seltenen gemeinsamen Fernsehabende, statt. Die Eltern scheinen vielmehr eher wenig Interesse daran zu haben, sich mit dem Fernsehumgang ihrer Söhne und Töchter auseinander zusetzen. (S. 9)

Die Mediennutzungspraxis der Kinder, die sich mit Hilfe der Einschaltquoten der Kinder identifizieren lässt, nehmen die Kasseler Medienpädagoginnen als Basis, um sozialkulturelle Funktionen von Fernsehangeboten zu diskutieren. Sie sehen Mediennutzung als aktives Sinnstiften (S. 3), das mit Hilfe der bevorzugten Fernsehprogramme Alltagsorientierung und Lebensgestaltung ermöglicht. Hier steht also der 〈Agency)-Aspekt im Argumentationsdreieck von Strukturen, Handeln (Agency) und kulturellen Praktiken im Vordergrund, der mit Hilfe der «Freizeitmustern» der SRTL-Studie «Kinderwelten 2000» theoretisch an die sozialkulturelle Fragmentierung angebunden wird. Die Ergebnisse zu Fernsehprogrammen, die auf Medienkompetenzförderung angelegt sind, weisen auf die Kompensation mangelnder sozialer Kontextualisierung, die auch Mikos, Töpper und Paus-Hasebrink et al. monieren. Nach den Kasseler Ergebnissen nutzen Kinder Sendungen zur Medienkompetenz, auch solche die komplex angelegt sind. Die Untersuchung identifiziert 
Sendungen in der Bandbreite von «Instruktion» («Regeln geben»), «diskursiven Umgang» («Regeln anwenden, Argumentationshilfen») sowie «Dekonstruktion und Neukonstruktion» («Regeln brechen ... individuelle Rezeption und Gestaltung») (S. 20 f.).

Ebenso auf Orientierung mit Relevanz für die Lebenspraxis der Kinder durch Fernsehprogramme ist die Untersuchung von Fleischer angelegt. Beratung im Fernsehen wird von den «zuschauenden Kindern als direktes Kommunikationsangebot verstanden» (S. 2). Auch hier besteht eine kompensatorische Chance, denn «teilweise» wird das «Beratungsangebotes als Orientierungsquelle» für "Kinder nur dann relevant, wenn andere Bezugspersonen Teil des Problems sind und daher nicht zu Rate gezogen werden können.» (S. 5) Trotzdem sehen Kinder in TV-Beratungsangeboten «weder Konkurrenz noch Ersatz für Eltern und Freunde.» (S. 6)

Die dänische Untersuchung von Johansen konzentriert sich vor allem auf das Feld der Handlungsmöglichkeiten, die sich für Kleinkinder in der Familie mit Fernsehprogramme eröffnen. Johansen stellt ihre Familienbeobachtungen in den direkten Zusammenhang mit neuen multimedialen Strukturen der Massenkommunikation, die Fernsehprogramme mit Kaufprodukten (Merchandising) verknüpft. Die Untersuchung von Kleinkindern in sieben Familien zeigt, dass Familien, obwohl sie das sehr unterschiedliche tun (S. 11) ihre Kinder in die Kulturwelt der Medien und des Konsums («contemporary consumer culture», S. 11) einführen. Sie tun dies, während die Kleinkinder zu regelmässigen TV-Nutzern werden, die ihre «klaren Präferenzen, Vorlieben und Abneigungen» (S. 8) haben. Dabei spielen die Kinder eine aktive Rolle, indem sie Bedeutungen herstellen («meaning-making processes», S. 1):

Toddlers ... involve themselves in contemporary media culture, and during the second and third year of their lives they become aware of many stylistic and narrative features of both programs and commercials, including different kind of trailers and internal advertising. ... television - not least aimed at the youngest viewers - works as a window for exhibition of a wide range of products related to the programs and for building the before mentioned brand-loyalty towards programs and channels. (S. 12)

\section{Was lässt sich als Fazit feststellen?}

In diesem Themenheft zum Kinderfernsehen zeichnet sich eine vorsichtige Ausrichtung auf die strukturellen Veränderungen der Massenkommunikation (Stichwort: Multimedia) und der sozialen Fragmentierung ab. Die in diesem Heft fokussierte Diskussion, an der sich keine Wissenschaftlerinnen und Wissenschafter aus der Sender nahen Forschung beteiligten, zeigt zudem die Veränderung der mit dem Fernsehen zusammenhängen Alltagpraxis der Kinder und Familien, wobei Kinder als die Bedeutung generierenden Akteure erscheinen. 


\section{Literaturnachweis}

Bachmair, Ben: Fernsehen in der Kinderkultur - Kinder verwenden es als Steinbruch. In: Zentralstelle Medien der Deutschen Bischofskonferenz, Gemeinschaftswerk der Evangelischen Publizistik 1998, S. 85-101.

Erlinger, Hans Dieter u. a. (Hrsg.): Handbuch des Kinderfernsehens. Konstanz (Ölschläger) 1995.

Erlinger, Hans Dieter, Stötzel, Dirk Ulf (Hrsg.): Geschichte des Kinderfernsehens in der Bundesrepublik Deutschland. Entwicklungsprozesse und Trends. Berlin (Wissenschaftsverlag Volker Spiess) 1991.

Giddens, Anthony: The Constitution of Society: Outline of the Theory of Structuration. University of California Press. 1984. Reprint edition (January 1, 1986).

Hall, Stuart (ed.): Representation. Cultural Representations and Signifying Practices. Sage. London 1997.

Kuchenbuch, Katharina: Die Fernsehnutzung von Kindern aus verschiedenen Herkunftsmilieus. In: Media Perspektiven Heft 1 2003, S. 2-11.

Paus-Haase, Ingrid: Vom Sesam-öffne-Dich des Vorschulbooms hin zur guten alten Tante des Kinderfernsehens der 90er Jahre. Die wechselvolle Geschichte der Sesamstrasse. In: Erlinger u. a. 1995, S. 177-192.

Schäfer, Albert: Spass an der Qualität. Zur Entwicklung des ZDF-Kinder und- und Jugendprogramms. In: Erlinger u. a. 1995, S.27-41.

Schmidbauer, Michael: Die Geschichte des Kinderfernsehens in der Bundesrepublik Deutschland. Eine Dokumentation. Schriftenreihe des Internationalen Zentralinstituts für das Jugend- und Bildungsfernsehen, Bd. 21. München (Saur) 1987.

Etienne Wenger: Communities of Practice. Learning, Meaning, and Identity. Cambridge, New York etc 1998. (Cambridge University Press).

Zentralstelle Medien der Deutschen Bischofskonferenz, Gemeinschaftswerk der Evangelischen Publizistik (Hrsg.): Debatte Kinderfernsehen. Analyse und Bewertung von TV-Programmen für Kinder. Berlin (Vistas) 1998. 